\title{
Investigations of the Lactate Minimum Test
}

Authors

Affiliation

\section{A. Johnson, G. R. Sharpe, P. I. Brown}

School of Science and Technology, Nottingham Trent University, Nottingham, United Kingdom

\section{Key words}

lactate kinetics

- test protocol

prior exercise

constant power accepted after revision

December 5, 2008

Bibliography

DOI 10.1055/s-0028-1119404

Published online:

February 6, 2009

Int J Sports Med 2009; 30:

448-454 (c) Georg Thieme

Verlag KG Stuttgart · New York ISSN 0172-4622

\section{Correspondence}

Dr. M. A. Johnson

School of Science and

Technology

Nottingham Trent University

Clifton Campus

Nottingham

United Kingdom

NG11 8NS

Tel.: +44/115/8483362

Fax: +44/115/848 6636

michael.johnson@ntu.ac.uk

\section{Abstract \\ $\nabla$}

We evaluated: the agreement between lactate minimum and maximal lactate steady state (MLSS) cycling powers (study 1); whether rates of change of blood lactate concentration during the lactate minimum test reflect that of constant power exercise (study 2); whether the lactate minimum power is influenced by the muscle groups used to elevate blood lactate concentration (study 3). Study 1: 32 subjects performed a lactate minimum test comprising a lactate elevation phase, recovery phase, and incremental phase (five 4 min stages); MLSS was subsequently determined. Study 2: 8 subjects performed a lactate minimum test and five 22 min constant power tests at the incremental phase exercise intensities. Study 3: 10 subjects performed two identical lactate minimum tests, except during the second test the lactate elevation phase comprised arm-cranking. Lactate minimum and MLSS powers demonstrated good agreement (mean bias $\pm 95 \%$ limits of agreement: $2 \pm 22 \mathrm{~W}$ ). Rates of change of blood lactate concentration during each incremental phase stage and corresponding constant power test did not correlate. Lactate minimum power was lowered when arm-cranking was used during the lactate elevation phase $(157 \pm 29$ vs. $168 \pm 21 \mathrm{~W} ; \mathrm{p}<0.05)$. The lactate elevation phase modifies blood lactate concentration responses during the incremental phase, thus good agreement between lactate minimum and MLSS powers seems fortuitous.

\section{Introduction}

$\nabla$

Tegtbur et al. [28] proposed the lactate minimum test as a method to predict maximal lactate steady state (MLSS). The test comprises three consecutive exercise phases: a lactate elevation phase, a short recovery phase, and an incremental phase in which with increasing intensity blood lactate concentration $\left(\left[\mathrm{lac}^{-}\right]_{\mathrm{B}}\right)$ decreases (net lactate clearance) to a nadir (the lactate minimum) and then increases (net lactate appearance). The $\left[\mathrm{lac}^{-}\right]_{\mathrm{B}}$ is determined by rates of lactate release into the interstitium or circulation, and consumption by adjacent or remote lactate-consuming oxidative muscle fibres or organs (the lactate shuttle [9]). The lactate minimum test has considerable value since MLSS is an important physiological determinant of endurance exercise performance $[17,21,24]$. Collectively, with the exception of one conflicting report [17], the literature suggests good agreement between lactate minimum and MLSS intensities [3,21,24,28] and the test has been recommended over other methods of MLSS prediction [27].

The ability to determine MLSS in one session has been used to advocate the use of the lactate minimum test [27]. However, Tegtbur et al. [28], and others thereafter $[3,17]$, used prior knowledge of subjects' training status to determine the incremental phase exercise intensities, whilst others $[13,21,23,24,26]$ have used pre-tests (time-trials and maximal incremental tests) to achieve the same end. Thus the test would be improved if the incremental phase exercise intensities could be resolved within the same test. Smith et al. [26] found that lactate minimum cycling power was independent of whether the lactate elevation phase comprised a maximal incremental ramp test or sprint exercise. Thus, modifying the original protocol of Tegtbur et al. [28] by replacing the short, high-intensity exercise of the lactate elevation phase with incremental exercise ought not to affect lactate minimum test validity but should also allow concurrent determination of maximal oxygen uptake. Therefore, in study 1 the aim was 
to evaluate the agreement between lactate minimum and MLSS cycling powers using a modified lactate minimum protocol in which the incremental phase intensities are based upon a maximal incremental ramp test performed in the lactate elevation phase.

Another feature used to promote the lactate minimum test is its "sound theoretical basis" [21]. This refers to the observations of Davis and Gass [11] on which Tegtbur et al. [28] based their protocol. Davis and Gass [11] introduced the innovation of examining $\Delta\left[\mathrm{lac}^{-}\right]_{\mathrm{B}} / \Delta \mathrm{t}$ (where $\mathrm{t}=$ time) during incremental exercise commencing with hyperlactaemia and proposed that such changes have "predictive value for steady-state work". However, this central premise remains unexplored and therefore in study 2 the aim was to examine whether $\Delta\left[\mathrm{lac}^{-}\right]_{\mathrm{B}} / \Delta \mathrm{t}$ during each stage of the incremental phase reflects $\Delta\left[\mathrm{lac}^{-}\right]_{\mathrm{B}} / \Delta \mathrm{t}$ measured during constant power exercise (after the initial transient change that occurs over the first 12 min of exercise [16]) at intensities identical to those performed during the incremental phase.

Prior exercise using the same muscle groups profoundly affects physiological responses to subsequent exercise, including reduced lactate production and release from the "primed" muscles $[5,10,15]$. Thus it seems likely that the lactate elevation phase will affect subsequent physiological responses and therefore the outcome of the test. To explore this issue the aim of study 3 was to examine whether $\Delta\left[\mathrm{lac}^{-}\right]_{B} / \Delta t$ and the lactate minimum cycling power during the incremental phase are influenced by the muscle groups used (leg cycling vs. arm-cranking) during the lactate elevation phase.

\section{Methods}

$\nabla$

Participants, equipment and measurements

Following local ethics committee approval, 50 non-smoking, recreationally active male subjects provided written informed consent to participate in the study. Subjects refrained from strenuous exercise during the $24 \mathrm{~h}$ preceding an exercise test. On test days subjects abstained from alcohol and caffeine and reported to the laboratory at least $2 \mathrm{~h}$ post-prandial. Successive tests were separated by at least $48 \mathrm{~h}$, but no more than 1 week, and were performed at a similar time of day.

Exercise was performed on an electromagnetically-braked cycle ergometer (Excalibur Sport, Lode, Groningen, The Netherlands) and also, during study 3, an electromagnetically-braked armcranking ergometer (Angio, Lode, Groningen, The Netherlands). The same self-selected cycling cadence was used throughout all tests. Arterialised venous blood samples were taken from a heated dorsal hand vein via an indwelling cannula [22] and analysed for $\left[\mathrm{lac}^{-}\right]_{\mathrm{B}}$ (P-GM7 MicroStat, Analox Instruments, London, UK). During the lactate elevation phase of study 1 , subjects wore a facemask (model 7940, Hans Rudolph, Kansas City, Missouri) and respiratory variables were measured breath-by-breath (Pulmolab EX670, Ferraris Respiratory Europe, Hertford, UK).

\section{Study 1 - agreement between lactate minimum and MLSS powers}

Subjects ( $\mathrm{n}=32$; age $29.9 \pm 6.9$ years, height $179.1 \pm 6.9 \mathrm{~cm}$, body mass $79.5 \pm 10.3 \mathrm{~kg}$ ) initially performed a lactate minimum test comprising 3 consecutive phases: (I) lactate elevation phase comprising maximal, incremental exercise; (II) 8 min recovery phase at $60 \mathrm{~W}$; and (III) incremental phase comprising five consecutive 4 min stages at intensities of $45,50,55,60$, and $65 \%$ of the maximum power ( $\dot{W} \max$ ) achieved during the lactate elevation phase. Changes in intensity during the incremental phase were based upon the original protocol of Tegtbur et al. [28] and pilot work was used to determine a range of intensities that would encompass the lactate minimum power in all subjects. Like previous studies $[13,17,24]$ the incremental phase comprised a fixed number of submaximal exercise stages rather than a maximal incremental exercise test, and the use of 5 stages was based upon the work of Jones and Doust [17]. The stage duration of $4 \mathrm{~min}$ was based upon the work of Tegtbur et al. [28]. Blood samples were taken from 11 subjects at the start of the incremental phase and every minute thereafter, and $\Delta\left[\mathrm{lac}^{-}\right]_{B} / \Delta t$ was taken as the gradient of a linear regression of $\left[\mathrm{lac}^{-}\right]_{\mathrm{B}}$ against time during each stage. Blood samples were taken from the remaining subjects at the end of each incremental phase stage. During the lactate elevation phase power increased every $15 \mathrm{~s}$ by a constant amount (8-10 W, depending upon the subject's training history) chosen so that exercise intolerance (cadence $<60 \mathrm{revs} \cdot \mathrm{min}^{-1}$ ) occurred in $\sim 10 \mathrm{~min}$. The final power defined $\dot{\mathrm{W}} \max$ and the highest oxygen uptake recorded over any 30 s period defined maximal oxygen uptake.

Maximal lactate steady state was resolved using 30 min constant power tests preceded by 3 min of cycling at $50 \%$ of the prescribed power. The first test was performed at lactate minimum power, and for subsequent tests power was adjusted by $\pm 2.5 \%$ until MLSS was verified. Blood samples were taken every $2 \mathrm{~min}$ from 16-30 min, inclusive, and MLSS power was defined as the highest power at which a positive gradient of a linear regression fitted through the plot of $\left[\mathrm{lac}^{-}\right]_{\mathrm{B}}$ against time was not observed [16].

Study 2 - comparison of the $\left[\mathrm{lac}^{-}\right]_{\mathrm{B}}$ response to the lactate minimum test and constant power cycling Subjects ( $n=8$; age $23.4 \pm 5.2$ years, height $180.4 \pm 6.4 \mathrm{~cm}$, body mass $79.9 \pm 5.5 \mathrm{~kg}$ ) initially performed a lactate minimum test (see study 1) with blood samples being taken at the start of the incremental phase and every minute thereafter. Subsequently, subjects performed, in random order and on different days, five 22 min cycling tests with blood samples being taken every 2 min from 0-22 min, inclusive. Cycling powers in each of the five tests corresponded to those used in the incremental phase, except that the initial $12 \mathrm{~min}$ of each test was always performed at $60 \%$ $\dot{\mathrm{W}}$ max to elevate $\left[\mathrm{lac}^{-}\right]_{\mathrm{B}}$. The magnitude of $\left[\mathrm{lac}^{-}\right]_{\mathrm{B}}$ is known to influence $\Delta\left[\operatorname{lac}^{-}\right]_{B} / \Delta t[11,26]$, thus it was essential that $\left[\operatorname{lac}^{-}\right]_{B}$ at the start of the lactate minimum incremental phase and corresponding constant power were closely matched; note also that pilot work showed that $\left[\mathrm{lac}^{-}\right]_{\mathrm{B}}$ remained unchanged from rest during square-wave exercise at 45 and 50\% $\dot{W}$ max. The initial 12 min allowed for the rapid, transient increase in $\Delta\left[\mathrm{lac}^{-}\right]_{B} / \Delta t$ from rest [16] and the total test duration ( $22 \mathrm{~min}$ ) was chosen as pilot work showed that this corresponded to the limit of exercise tolerance when cycling at 65\% $\mathbf{W}$ max. During constant power exercise $\Delta\left[\mathrm{lac}^{-}\right]_{\mathrm{B}} / \Delta \mathrm{t}$ was taken as the gradient of a linear regression of $\left[\mathrm{lac}^{-}\right]_{\mathrm{B}}$ against time (14-22 min, inclusive). Our analysis of $\Delta\left[\mathrm{lac}^{-}\right]_{\mathrm{B}} / \Delta \mathrm{t}$ was therefore performed over an $8 \mathrm{~min}$ period: this was the maximum possible time allowed by our subjects' ability to tolerate cycling at $65 \% \dot{\mathrm{W}} \max (22 \mathrm{~min})$ and the need to allow the initial transient increase in $\Delta\left[\mathrm{lac}^{-}\right]_{\mathrm{B}} / \Delta \mathrm{t}$ during the initial $12 \mathrm{~min}$ of exercise [16]. 

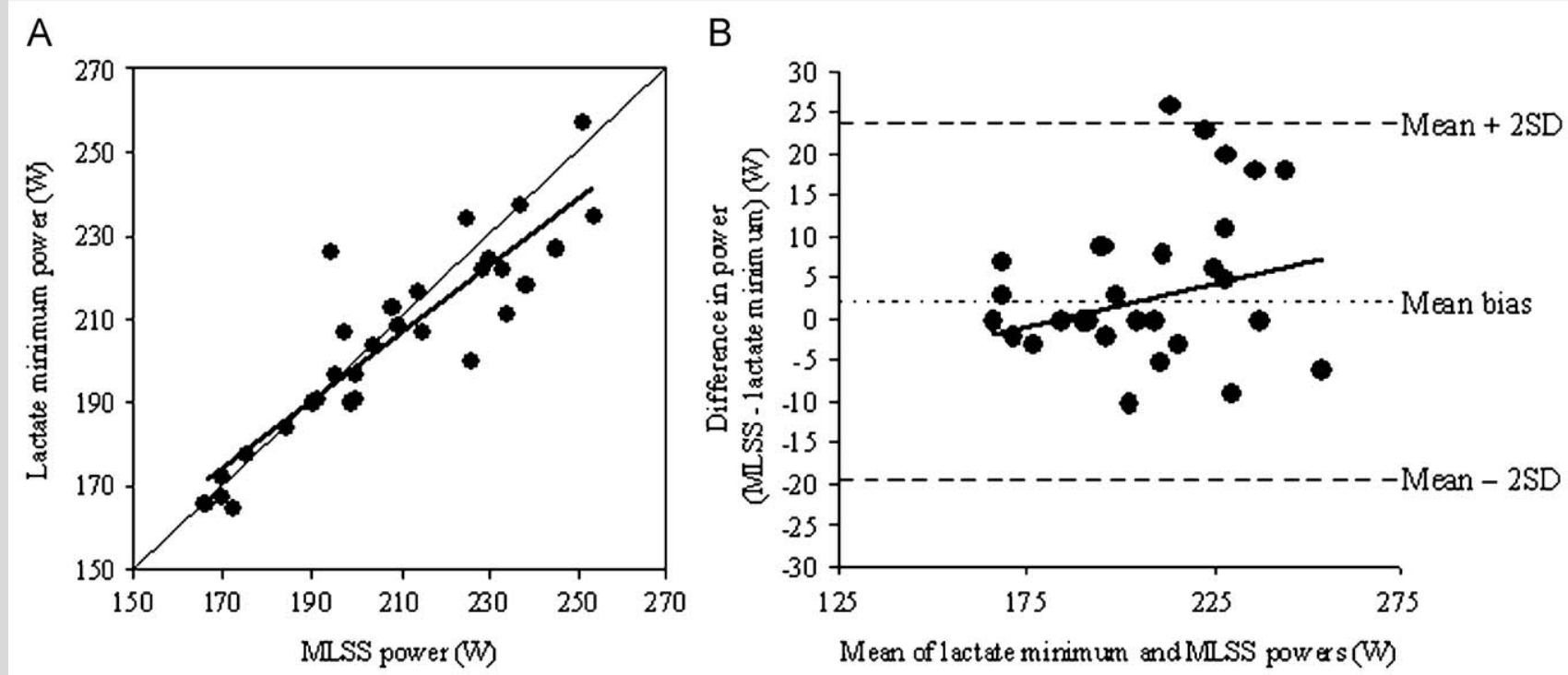

Fig. 1 A: Relationship between lactate minimum and MLSS powers, showing the line of identity. B: Agreement between lactate minimum and MLSS powers, showing the mean bias and $95 \%$ limits of agreement. Individual data are shown.

Study 3 - effect of muscle groups used during the lactate elevation phase

Subjects ( $\mathrm{n}=10$; age $23.5 \pm 5.5$ years, height $178.8 \pm 6.9 \mathrm{~cm}$, body mass $78.0 \pm 7.5 \mathrm{~kg}$ ) initially performed a lactate minimum test (see study 1 , hereafter termed $\mathrm{LM}_{\mathrm{LEG}}$ ). On a separate day, subjects repeated $\mathrm{LM}_{\mathrm{LEG}}$, except that the lactate elevation phase comprised maximal incremental arm-cranking exercise (hereafter termed $\mathrm{LM}_{\mathrm{ARM}}$ ). The centre of the arm-crank shaft was aligned to shoulder level and subjects were seated so that the elbow was slightly flexed when the hand was most distal. Following $15 \mathrm{~s}$ unloaded exercise, power was increased every $15 \mathrm{~s}$ by either 4 or $5 \mathrm{~W}$ up to the limit of exercise tolerance (cadence $<40$ revs $\left.\cdot \min ^{-1}\right)$. Subjects then transferred to the adjacentlypositioned cycle ergometer and, following the recovery phase $(8 \mathrm{~min}$ at $60 \mathrm{~W}$ ), repeated the incremental phase (using identical cycling powers) performed in $\mathrm{LM}_{\mathrm{LEG}}$. Thus, $\mathrm{LM}_{\mathrm{LEG}}$ always preceded $\mathrm{LM}_{\mathrm{ARM}}$. Blood samples were taken at the start of the incremental phase and every minute thereafter.

\section{Data analyses}

Data analyses were performed using SPSS (version 15). The lactate minimum power was determined from the zero gradient tangent to a cubic spline function fitting the $\left[\mathrm{lac}^{-}\right]_{B}$ (measured at the end of each stage) vs. power data. Data were analysed using repeated measures ANOVA and paired t-tests where appropriate. Agreement between variables was assessed using a Bland-Altman plot [7], along with the calculated bias $\pm 95 \%$ limits of agreement. Pearson product-moment correlation coefficients ( $\mathrm{r}$ ) were determined to assess the relationship between variables. Results are reported as mean \pm SD unless otherwise stated. Statistical significance was set at $\mathrm{p}<0.05$.

\section{Results}

$\nabla$

The $\left[\operatorname{lac}^{-}\right]_{\mathrm{B}}$ profile during the incremental phase was well described by the cubic spline function $\left(r^{2}=0.94\right.$ and 0.98 in study 1 and 2 respectively, and in study $3, \mathrm{r}^{2}=0.95$ and 0.99 for $\mathrm{LM}_{\mathrm{LEG}}$ and $\mathrm{LM}_{\mathrm{ARM}}$, respectively). Also, in each study lactate minimum power was correlated with $\dot{\mathrm{W}} \max (\mathrm{r}=0.97$ and 0.98 in study 1 and 2, respectively, and in study $3, \mathrm{r}=0.93$ for $\left.\mathrm{LM}_{\mathrm{LEG}}\right)(\mathrm{p}<0.01)$.

\section{Study 1 - agreement between lactate minimum and MLSS powers}

The $\dot{W} \max$ and maximal oxygen uptake were $379 \pm 42 \mathrm{~W}$ and $3.99 \pm 0.58 \mathrm{~L} \cdot \mathrm{min}^{-1}$, respectively. The lactate minimum power $(205 \pm 22 \mathrm{~W} ; 54.2 \pm 1.5 \%$ Wmax $)$ was not different from MLSS power $(208 \pm 25 \mathrm{~W} ; 54.9 \pm 3.6 \% \dot{\mathrm{W} m a x})$, with which it was correlated $(r=0.89, p<0.01)$ ( $\odot$ Fig. $1 \mathrm{~A})$. Although the gradient of the regression line in 0 Fig. $\mathbf{1 A}(0.804)$ is different from 1 $(p<0.05)$, this does not imply a lack of agreement between the two measurements $[1,7]$. Indeed, 0 Fig. 1B, which shows the difference between lactate minimum and MLSS powers against their mean (Bland-Altman plot) [7], along with the bias $\pm 95 \%$ limits of agreement $(2 \pm 22 \mathrm{~W})$ for the comparison between the two variables, indicates good agreement between lactate minimum and MLSS powers (for further commentary see Discussion). Furthermore, the gradient of the regression line in - Fig. 1B $(0.105)$ is not different from zero $(p=0.245)$, which indicates uniformity of systematic error across the range of measurements studied [1]. The difference between lactate minimum and MLSS powers was correlated with MLSS power $(\mathrm{r}=0.42, \mathrm{p}<0.05)$.

Where $\Delta\left[\mathrm{lac}^{-}\right]_{\mathrm{B}} / \Delta \mathrm{t}$ during each stage of the incremental phase was determined $(\mathrm{n}=11)$, the power at which $\Delta\left[\mathrm{lac}^{-}\right]_{\mathrm{B}} / \Delta \mathrm{t}=0$ $\left(220 \pm 21 \mathrm{~W} ; 58.5 \pm 5.7 \%\right.$ Wmax; $\left.\mathrm{r}^{2}=0.90 \pm 0.12\right)$ was greater $(\mathrm{p}<0.01)$ than lactate minimum $(203 \pm 22 \mathrm{~W} ; 53.7 \pm 1.4 \% \mathrm{~W}$ max) and MLSS powers (208 $\pm 26 \mathrm{~W} ; 54.7 \pm 2.7 \%$ Wmax). The bias $\pm 95 \%$ limits of agreement for the comparison of the power at which $\Delta\left[\mathrm{lac}^{-}\right]_{\mathrm{B}} / \Delta \mathrm{t}=0$ and MLSS power was $12 \pm 43 \mathrm{~W}$.

Study 2 - comparison of $\Delta\left[\mathrm{lac}^{-}\right]_{B} / \Delta$ t during the lactate minimum test and constant power cycling

The $\dot{W}$ max and lactate minimum power were $363 \pm 42 \mathrm{~W}$ and $193 \pm 24 \mathrm{~W}(52.8 \pm 1.2 \%$ Wmax $)$, respectively. During constant power exercise at $50 \% \dot{W} \max$, the $\left[\mathrm{lac}^{-}\right]_{\mathrm{B}}$ at $14 \mathrm{~min}$ 

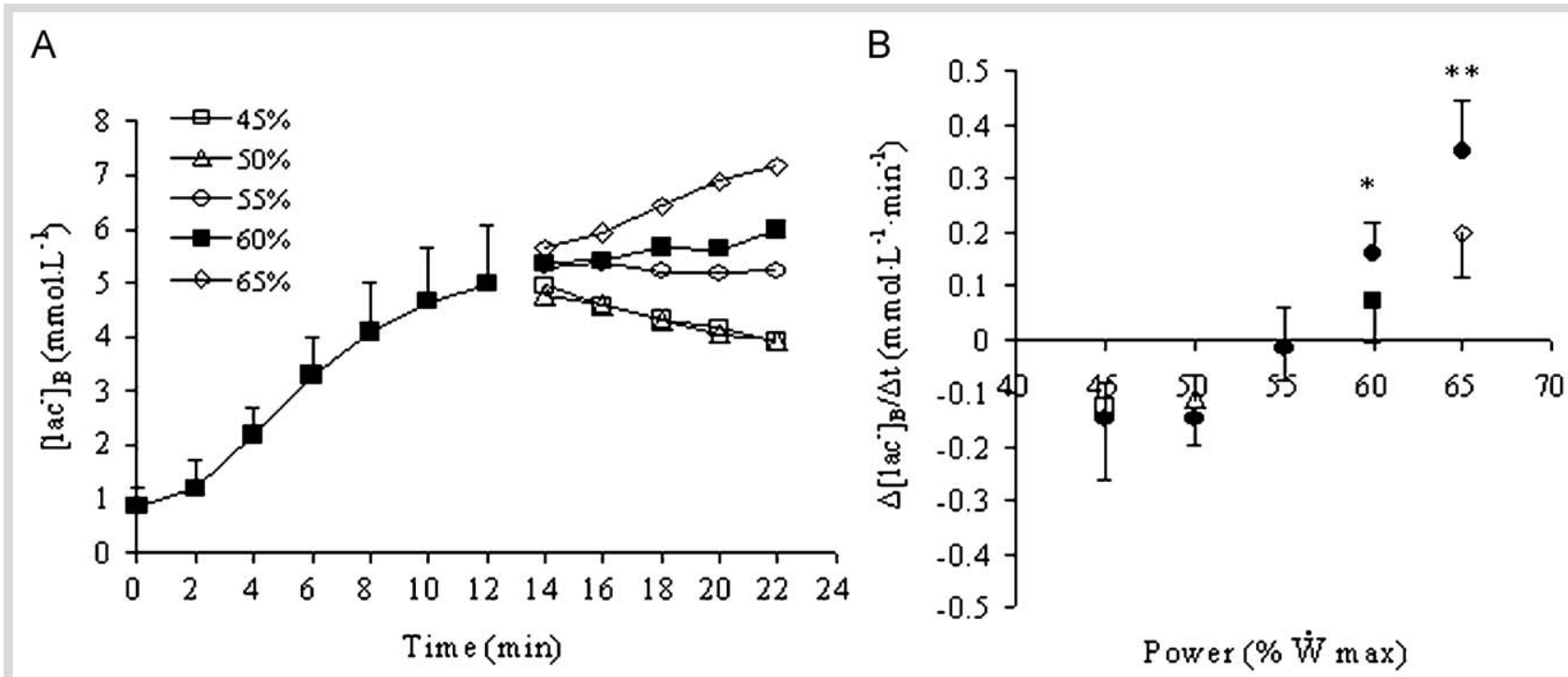

Fig. 2 A: Blood lactate concentration ([lac $\left.{ }^{-}\right]_{B}$ ) during constant power exercise. B: $\Delta\left[\text { lac }^{-}\right]_{B} / \Delta t$ during constant power exercise (see panel 'A' for symbols) and each stage of the lactate minimum incremental phase $(\bullet)$. Note that $\Delta\left[\mathrm{lac}^{-}\right]_{\mathrm{B}} / \Delta \mathrm{t}$ for constant power exercise reflects that measured over $14-22 \mathrm{~min}$ in ' $A$ '. Values are mean $\pm S D$, except in 'A' where for clarity error bars are shown only for the initial 12 min of exercise. Difference between trials, ${ }^{*} p<0.05$, ${ }^{* *} \mathrm{p}<0.01$.

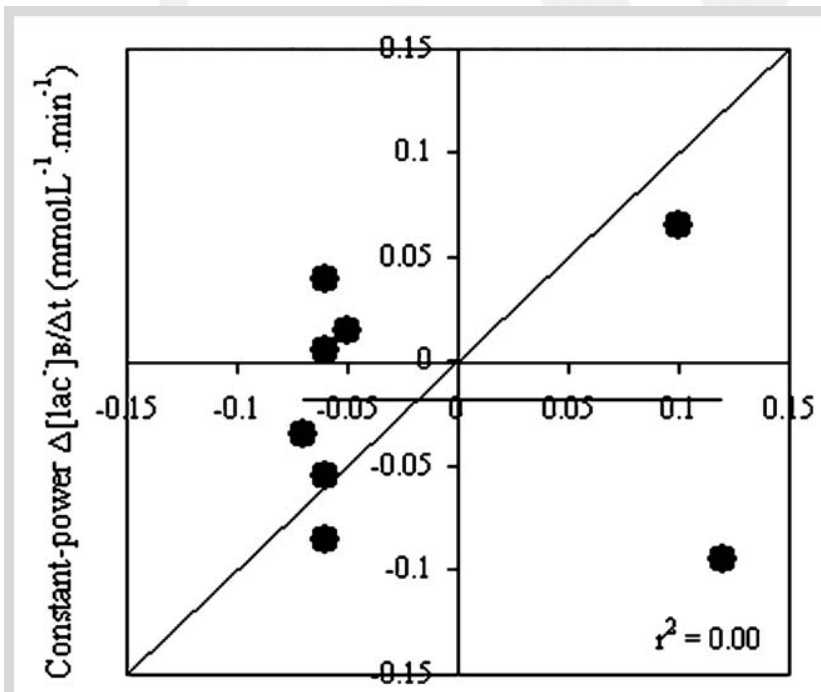

Incremental phase $\Delta[\mathrm{lac}]_{\mathrm{B}} / \Delta \mathrm{t}\left(\mathrm{mmol} \cdot \mathrm{L}^{-1} \cdot \mathrm{min}^{-1}\right)$

Fig. 3 Individual rates of change of blood lactate concentration $\left(\Delta\left[\mathrm{lac}^{-}\right]_{\mathrm{B}} / \Delta \mathrm{t}\right)$ during exercise at $55 \% \dot{\mathrm{W}}$ max during the lactate minimum incremental phase and constant power exercise. Line of identity is shown.

$\left(4.8 \pm 1.0 \mathrm{mmol} \cdot \mathrm{L}^{-1}\right)$ (see $\odot$ Fig. $2 \mathrm{~A}$ ) was lower than that measured at the commencement of $50 \%$ Wmax during the incremental phase $\left(5.5 \pm 1.2 \mathrm{mmol} \cdot \mathrm{L}^{-1}\right)$. There were no such differences at the other 4 intensities.

Compared to constant power exercise, $\Delta\left[\mathrm{lac}^{-}\right]_{\mathrm{B}} / \Delta \mathrm{t}$ was greater during the incremental phase at $60 \%(\mathrm{p}<0.05)$ and $65 \% \dot{\mathrm{W}} \max$ $\left(\mathrm{p}<0.01\right.$ ) ( 0 Fig. 2B). The power at which $\Delta\left[\mathrm{lac}^{-}\right]_{\mathrm{B}} / \Delta \mathrm{t}=0$ during the incremental phase $(195 \pm 24 \mathrm{~W} ; 53.7 \pm 1.9 \%$ Wmax; $\mathrm{r}^{2}=0.84 \pm 0.10$ ) was not different from that determined in constant power exercise $(200 \pm 28 \mathrm{~W}$; $55.1 \pm 3.3 \%$ Wंmax; $\left.\mathrm{r}^{2}=0.91 \pm 0.06\right)$. However, $\Delta\left[\mathrm{lac}^{-}\right]_{\mathrm{B}} / \Delta \mathrm{t}$ during each incremental phase stage and corresponding constant power test did not correlate (at each \% $\dot{W} \max , \mathrm{r}$ values were: $45 \%=0.07,50 \%=0.00$, $55 \%=-0.03,60 \%=0.14$, and $65 \%=0.63$ ) (e.g., o Fig. 3 ).

\section{Study 3 - effect of muscle groups used during the} lactate elevation phase

The duration of the lactate elevation phase during $\mathrm{LM}_{\mathrm{ARM}}$ $(9.13 \pm 1.62 \mathrm{~min})$ was not different from that in $\mathrm{LM}_{\mathrm{LEG}}$ (9.49 $\pm 0.74 \mathrm{~min})$, although $\dot{\mathrm{W}} \max$ was expectedly lower during $\mathrm{LM}_{\text {ARM }}(161 \pm 44$ vs. $317 \pm 44 \mathrm{~W})(\mathrm{p}<0.01)$. All subjects demonstrated a U-shaped $\left[\mathrm{lac}^{-}\right]_{\mathrm{B}}$ vs. power profile during the incremental phase of $\mathrm{LM}_{\mathrm{LEG}}$ and the lactate minimum power was $168 \pm 21 \mathrm{~W}(53.3 \pm 2.9 \%$ Wmax $)$. Conversely, a clear U-shaped profile was not observed in $\mathrm{LM}_{\mathrm{ARM}}(\odot$ Fig. $4 \mathrm{~A}$ ), and in one subject a lactate minimum power could not be determined because $\left[\mathrm{lac}^{-}\right]_{\mathrm{B}}$ increased linearly $\left(\mathrm{r}^{2}=1\right)$. In the remaining subjects lactate minimum power during $\operatorname{LM}_{\mathrm{ARM}}(157 \pm 29 \mathrm{~W})$ was lower than that in $\operatorname{LM}_{\mathrm{LEG}}(\mathrm{p}<0.05)$. The $\left[\mathrm{lac}^{-}\right]_{B}$ at the end of each stage of the incremental phase was not different between $\mathrm{LM}_{\mathrm{LEG}}$ and $\mathrm{LM}_{\mathrm{ARM}}$, although at $60 \% \mathrm{~W} \max$ there was a trend $(\mathrm{p}=0.055)$ for $\left[\mathrm{lac}^{-}\right]_{B}$ to be higher during $\mathrm{LM}_{\mathrm{ARM}}$.

The power at which $\Delta\left[\mathrm{lac}^{-}\right]_{\mathrm{B}} / \Delta \mathrm{t}=0$ in $\operatorname{LM}_{\mathrm{LEG}}(175 \pm 24 \mathrm{~W}$; $\left.55.4 \pm 2.6 \% \dot{W} \max ; r^{2}=0.85 \pm 0.13\right)$ was greater than that in $\operatorname{LM}_{\text {ARM }}\left(157 \pm 31 \quad\right.$ W; $\left.49.4 \pm 6.3 \% \quad \dot{W} \max ; \quad \mathrm{r}^{2}=0.88 \pm 0.12\right)$ ( $\triangle$ Fig. 4B). There was a trial $\times$ stage interaction effect for $\Delta\left[\mathrm{lac}^{-}\right]_{\mathrm{B}} / \Delta \mathrm{t}(\mathrm{p}<0.01)$, and differences were observed between $\mathrm{LM}_{\mathrm{LEG}}$ and $\mathrm{LM}_{\mathrm{ARM}}$ at $50 \%(\mathrm{p}<0.01)$ and $60 \%(\mathrm{p}<0.05) \mathrm{W}$ max.

\section{Discussion}

$\nabla$

The main findings of the present study were threefold: (I) there was good agreement between lactate minimum and MLSS powers using the modified lactate minimum protocol; (II) $\Delta\left[\operatorname{lac}^{-}\right]_{\mathrm{B}} / \Delta \mathrm{t}$ during each stage of the incremental phase did not reflect $\Delta\left[\mathrm{lac}^{-}\right]_{\mathrm{B}} / \Delta \mathrm{t}$ during constant power exercise; and (III) the $\left[\mathrm{lac}^{-}\right]_{\mathrm{B}}$ 

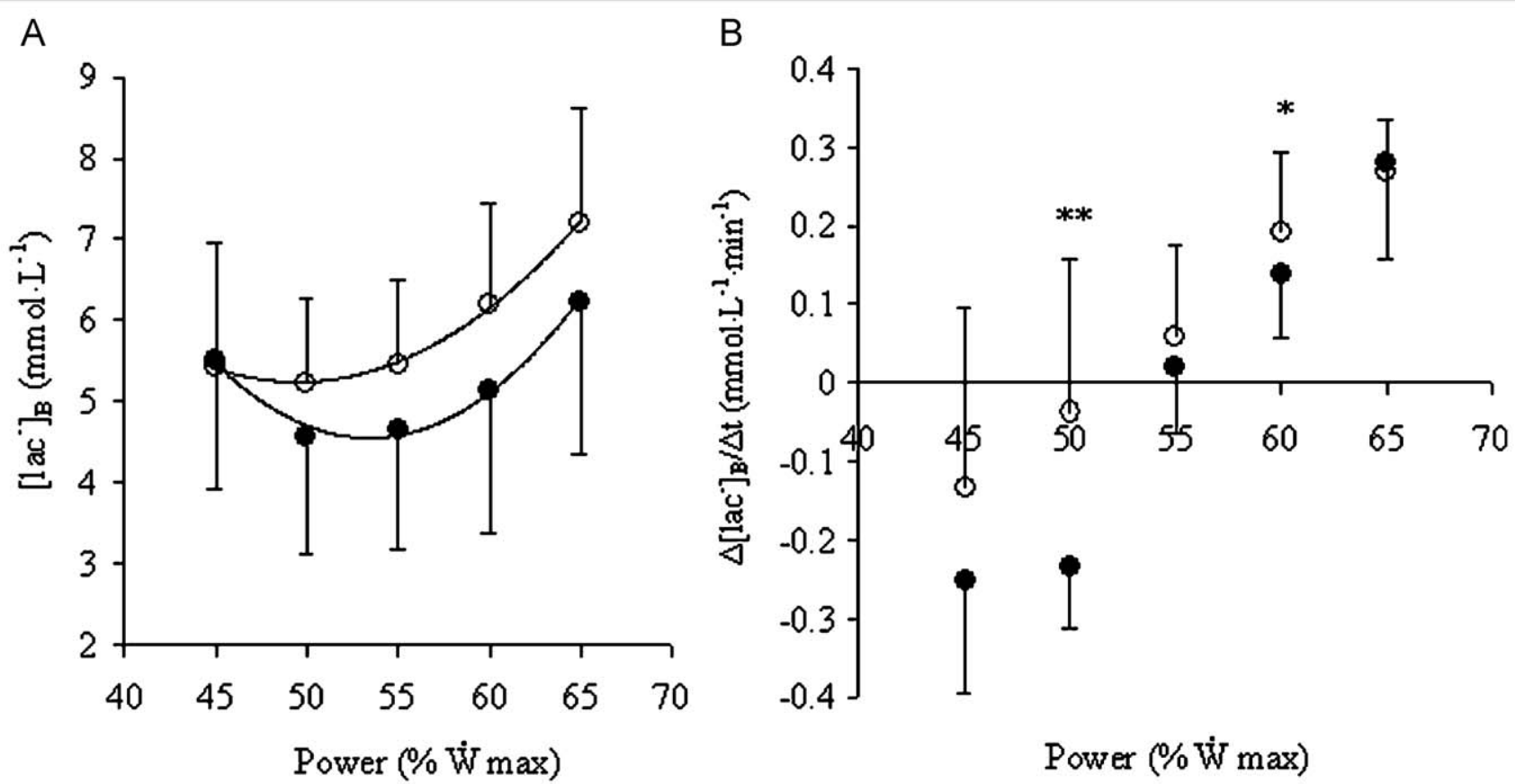

\section{Power (\% Wं max)}

Power (\% Wं max)

Fig. 4 A: Blood lactate concentration $\left(\left[\operatorname{lac}^{-}\right]_{\mathrm{B}}\right)$ at the end of each lactate minimum incremental phase stage. B: $\Delta\left[\operatorname{lac}^{-}\right]_{\mathrm{B}} / \Delta \mathrm{t}$ during each lactate minimum incremental phase stage. $\operatorname{LM}_{\mathrm{LEG}}(\bullet), \operatorname{LM}_{\text {ARM }}(0)$. Values are mean $\pm \mathrm{SD}$. Difference between trials, ${ }^{*} \mathrm{p}<0.05,{ }^{* *} \mathrm{p}<0.01$.

profile of the incremental phase was dependent upon whether the lactate elevation phase was performed using the same or different muscle groups.

Given the resolution with which MLSS is typically determined (10-20 W) [27], the mean difference and 95\% limits of agreement $(2 \pm 22 \mathrm{~W})$ for the comparison between lactate minimum and MLSS powers indicates that our modified lactate minimum test provides an acceptable estimate of MLSS power. These findings support other work showing the lactate minimum to be a valid predictor of MLSS [21,24], although others report conflicting evidence [17]. Note also that statistical power analysis revealed that, in our study, the minimum detectable difference (based upon our sample size $(n=32)$, an alpha level of 0.05 , and power of 0.8 ) between lactate minimum and MLSS powers was $6 \mathrm{~W}$. This corresponds closely to our MLSS resolution and therefore may be considered the minimum difference of practical significance [27]. In addition to estimating MLSS, our lactate minimum protocol also allows determination of maximal oxygen uptake and does not require detailed knowledge of subjects' training status. This is an improvement on previously described lactate minimum protocols in which prescription of incremental phase exercise intensities has required either separate testing or familiarity with subjects' training status (see Introduction). Furthermore, determining both maximal oxygen uptake and MLSS provides a more complete assessment of training status and training programme effectiveness. Our lactate minimum protocol was also well tolerated by subjects of varied training status (MLSS range: 166-253 W), which allayed our initial concerns that combining a maximal incremental test with the incremental phase may be too demanding for less-trained subjects.

Absolute $\left[\mathrm{lac}^{-}\right]_{\mathrm{B}}$ values and "thresholds" in ventilatory, pulmonary gas exchange, and $\left[\mathrm{lac}^{-}\right]_{\mathrm{B}}$ responses to incremental exercise have also been used to predict MLSS, although the validity of such protocols is poorly documented. It is well recognised, however, that the large inter-individual variation in $\left[\mathrm{lac}^{-}\right]_{\mathrm{B}}$ at MLSS
(3-10 $\mathrm{mmol} \cdot \mathrm{L}^{-1}[21]$,) precludes MLSS prediction from absolute $\left[\operatorname{lac}^{-}\right]_{\mathrm{B}}$ values $[2,17,27]$. Conversely, the "lactate turnpoint" may provide a good estimate of MLSS $[2,18]$, although Smith and Jones [25] report significant over- or under-estimation in individual subjects. The validity of the individual anaerobic threshold test is also variable, providing close estimates of MLSS in cycling [29] and running [4], but overestimating MLSS in rowing [6]. The respiratory compensation point often overestimates MLSS [12,20], whereas Laplaud et al. [20] suggest that MLSS corresponds to the intensity during incremental exercise at which the respiratory exchange ratio $=1.00$. However, the lack of resolution in MLSS determination in this study (5\% W max, 16 W) could have masked significant disagreement between the predicted and the "true" MLSS. When viewed collectively, no single test can accurately determine MLSS, and comparison of tests is complicated by inter-study differences in exercise modalities and protocols, participants, and MLSS determination methods. Unlike many of the aforementioned protocols, however, the lactate minimum test provides a reliable, objective MLSS estimate $[21,26]$ that is insensitive to changes in muscle glycogen stores [28]. It is therefore an attractive option amongst a plethora of single-test methods to predict MLSS. However, the level of exertion required during the lactate minimum test renders the protocol impractical for clinical populations [27]; threshold determinations from submaximal exercise are more appropriate under these circumstances.

We avoided basing our MLSS criterion on absolute increases in $\left[\mathrm{lac}^{-}\right]_{\mathrm{B}}$ over time (see MacIntosh et al. [21] for a discussion) since this results in dissimilar relative physiological stress due to the large inter-individual variation in $\left[\mathrm{lac}^{-}\right]_{\mathrm{B}}$ at $\operatorname{MLSS}\left(3-10 \mathrm{mmol} \cdot \mathrm{L}^{-1}\right.$ [21],). We also used relatively small step changes at an intensity of $\pm 2.5 \%(\sim 4-6 \mathrm{~W})$, which allows greater resolution of MLSS than those studies using increments of $4-5 \%$ or $\sim 10-20 \mathrm{~W}$ (reviewed in Svedahl and MacIntosh [27]). 
Lactate minimum and MLSS agreement decreased as these cycling powers increased. Variability in MLSS prediction accuracy has been reported previously $[3,21]$, and though the reason(s) for this remains unclear, MacIntosh et al. [21] suggest that prior training-related fatigue may affect the outcome of the lactate minimum test. Although our subjects refrained from exercise for at least $24 \mathrm{~h}$ prior to each test it remains possible that residual effects of the increased training volume associated with higher MLSS powers adversely affected the MLSS prediction.

The findings of study 2 show that $\Delta\left[\mathrm{lac}^{-}\right]_{\mathrm{B}} / \Delta \mathrm{t}$ during the incremental phase and constant power exercise did not correlate. To our knowledge, we are the first to report such data and they challenge the proposition of Davis and Gass [11] that $\Delta\left[\mathrm{lac}^{-}\right]_{\mathrm{B}} / \Delta \mathrm{t}$ during incremental exercise commencing with hyperlactaemia has "predictive value for steady state work". Since the magnitude of $\left[\mathrm{lac}^{-}\right]_{\mathrm{B}}$ partly determines $\Delta\left[\mathrm{lac}^{-}\right]_{\mathrm{B}} / \Delta \mathrm{t}[11,26]$, we strived to match $\left[\mathrm{lac}^{-}\right]_{\mathrm{B}}$ at the start of each incremental phase stage and corresponding constant power. This was achieved at all powers except $50 \% \dot{W} \max$ and we feel that this difference is unlikely to explain the absence of a correlation for $\Delta\left[\mathrm{lac}^{-}\right]_{\mathrm{B}} / \Delta \mathrm{t}$ between the two conditions. Thus, our findings not only challenge the theoretical underpinning that has been used to promote the lactate minimum test [21], but they also suggest that the dynamics of the lactate shuttle during the incremental phase differ from that in constant power exercise at equivalent intensities. Furthermore, lactate minimum and MLSS powers have different mathematical definitions: $\Delta\left[\mathrm{lac}^{-}\right]_{\mathrm{B}} / \Delta \mathrm{P}=0$ (where $\mathrm{P}=$ cycling power) and $\Delta\left[\mathrm{lac}^{-}\right]_{\mathrm{B}} / \Delta \mathrm{t}=0$, respectively, and the cycling powers corresponding to these solutions were different (see study 1 ). Also, paradoxically the agreement with MLSS power was stronger for $\Delta\left[\mathrm{lac}^{-}\right]_{\mathrm{B}} / \Delta \mathrm{P}=0$ (lactate minimum) compared to $\Delta\left[\mathrm{lac}^{-}\right]_{\mathrm{B}} / \Delta \mathrm{t}=0$. These findings support previous conjecture [17] that good agreement between lactate minimum and MLSS powers partly reflects a fortuitous artefact of the protocol design.

The findings of study 3 are the first to show that the lactate minimum test is strongly influenced by the muscle groups used during the lactate elevation phase. The $\mathrm{LM}_{\mathrm{ARM}}$ protocol precluded a U-shaped $\left[\mathrm{lac}^{-}\right]_{\mathrm{B}}$ profile during the incremental phase, which resulted in consistently greater values for $\Delta\left[\mathrm{lac}^{-}\right]_{\mathrm{B}} / \Delta \mathrm{t}$, and lowered the lactate minimum power compared to $\mathrm{LM}_{\mathrm{LEG}}$. These findings support previous observations of increased $\Delta\left[\mathrm{lac}^{-}\right]_{\mathrm{B}} / \Delta \mathrm{t}$ during cycling exercise preceded by heavy exercise using different compared to the same muscle groups $[8,14]$. Since $\Delta\left[\mathrm{lac}^{-}\right]_{\mathrm{B}} /$ $\Delta t$ depends upon the magnitude of $\left[\operatorname{lac}^{-}\right]_{\mathrm{B}}[11,26]$ we ensured that this was equal at the beginning of the incremental phase of $\mathrm{LM}_{\mathrm{ARM}}$ and $\mathrm{LM}_{\mathrm{LEG}}$, and thus attribute the different responses to the effects of using the same or different muscle groups in the lactate elevation phase. The influence of the lactate elevation phase may partially explain why $\Delta\left[\mathrm{lac}^{-}\right]_{\mathrm{B}} / \Delta \mathrm{t}$ during the incremental phase and constant power exercise failed to correlate (study 2). The lactate elevation phase may well "prime" the working muscles prior to the incremental phase, thus reducing metabolic inertia (i.e., the delay in oxidative metabolism at the onset of exercise [15]). Such priming only occurs when prior exercise involves the same muscle groups $[8,14,19]$ and is predominantly manifest early in the subsequent exercise bout [10]. This is consistent with the data shown in $\bullet$ Fig. 3, where differences in $\Delta\left[\mathrm{lac}^{-}\right]_{\mathrm{B}} / \Delta \mathrm{t}$ between $\mathrm{LM}_{\mathrm{LEG}}$ and $\mathrm{LM}_{\mathrm{ARM}}$ were manifest during the initial stages of the incremental phase. Therefore, the lactate elevation phase of $\mathrm{LM}_{\mathrm{LEG}}$, unlike that of $\mathrm{LM}_{\mathrm{ARM}}$, probably increased the aerobic contribution to the energy demand during the initial stages of the incremental phase, thus resulting in less lactate accumulation and greater lactate oxidation $[5,10,15]$. This is supported by Simões et al. [24] who compared responses to the incremental phase with and without prior exercise and found elevated oxygen uptake and reduced respiratory exchange ratio in the former condition.

In summary, our modified lactate minimum test protocol provides valid measures of both maximal oxygen uptake and MLSS. However, $\Delta\left[\mathrm{lac}^{-}\right]_{\mathrm{B}} / \Delta \mathrm{t}$ during the incremental phase fails to reflect that of constant power exercise, possibly because the dynamics of the lactate shuttle, and therefore the $\left[\mathrm{lac}^{-}\right]_{\mathrm{B}}$ response, are modified by the high-intensity exercise performed in the lactate elevation phase. Consequently, good agreement between lactate minimum and MLSS powers seems fortuitous and requires a physiological explanation different from that based on the proposition of Davis and Gass [11].

\section{Acknowledgements}

$\nabla$

We thank Dr. Nigel Gleeson for proofreading the manuscript and we acknowledge financial support from Nottingham Trent University.

\section{References}

1 Atkinson G, Davison RCR, Nevill AM. Performance characteristics of gas analysis systems: what we know and what we need to know. Int J Sports Med 2005; 26: 2-10

2 Aunola S, Rusko H. Does anaerobic threshold correlate with maximal lactate steady state? J Sports Sci 1992; 10: 309-323

3 Bacon L, Kern M. Evaluating a test protocol for predicting maximum lactate steady state. J Sports Med Phys Fitness 1999; 39: 300-308

4 Baldari C, Guidetti L. A simple method for individual anaerobic threshold as predictor of max lactate steady state. Med Sci Sports Exerc 2000; 32: 1798-1802

5 Bangsbo J, Krustrup P, González-Alonso J, Saltin B. ATP production and efficiency of human skeletal muscle during intense exercise: effect of previous exercise. Am J Physiol Endocrinol Metab 2001; 280: 956-964

6 Beneke R. Anaerobic threshold, individual anaerobic threshold, and maximal lactate steady state in rowing. Med Sci Sports Exerc 1995; 27: 863-867

7 Bland JM, Altman DG. Statistical methods for assessing agreement between two methods of clinical measurement. Lancet 1986; 1: 307-310

8 Bohnert B, Ward SA, Whipp BJ. Effects of prior arm exercise on pulmonary gas exchange kinetics during high-intensity leg exercise in humans. Exp Physiol 1998; 83: 557-570

9 Brooks GA. Intra- and extra-cellular lactate shuttles. Med Sci Sports Exerc 2000; 32: 790-799

10 Campbell-O'Sullivan SP, Constantin-Teodosiu D, Peirce N, Greenhaff PL. Low intensity exercise in humans accelerates mitochondrial ATP production and pulmonary oxygen kinetics during subsequent more intense exercise. J Physiol 2002; 538: 931-939

11 Davis HA, Gass GC. Blood lactate concentrations during incremental work before and after maximum exercise. Br J Sports Med 1979; 13: 165-169

12 Dekerle J, Baron B, Dupont L, Vanvelcenaher J, Pelayo P. Maximal lactate steady state, respiratory compensation threshold and critical power. Eur J Appl Physiol 2003; 89: 281-288

13 Denadai BS, Higino WP. Effect of the passive recovery period on the lactate minimum speed in sprinters and endurance runners. J Sci Med Sport 2004; 7: 488-496

14 Fukuba $Y$, Hayashi $N$, Koga S, Yoshida $Y . \mathrm{VO}_{2}$ kinetics in heavy exercise is not altered by prior exercise with a different muscle group. J Appl Physiol 2002; 92: 2467-2474

15 Gurd BJ, Peters SJ, Heigenhauser GJF, LeBlanc PJ, Doherty TJ, Paterson $\mathrm{DH}$, Kowalchuk JM. Prior heavy exercise elevates pyruvate dehydrogenase activity and speeds $\mathrm{O} 2$ uptake kinetics during subsequent moderate-intensity exercise in healthy young adults. J Physiol 2006; 577: 985-996 
16 Johnson MA, Sharpe GR, MacConnell AK. Maximal voluntary hyperpnoea increases blood lactate concentration during exercise. Eur J Appl Physiol 2006; 96: 600-608

17 Jones AM, Doust JH. The validity of the lactate minimum test for determination of the maximal lactate steady state. Med Sci Sports Exerc 1998; 30: 1304-1313

18 Kilding AE, Jones AM. Validity of a single-visit protocol to estimate the maximum lactate steady state. Med Sci Sports Exerc 2005; 37: 17341740

19 Koppo K, Jones AM, Bouckaert J. Effect of prior heavy arm and leg exercise on $\mathrm{VO}_{2}$ kinetics during heavy leg exercise. Eur J Appl Physiol 2003; 88: 593-600

20 Laplaud D, Guinot M, Favre-Juvin A, Flore P. Maximal lactate steady state determination with a single incremental test exercise. Eur J Appl Physiol 2006; 96: 446-452

21 MacIntosh BR, Esau S, Svedahl K. The lactate minimum test for cycling: estimation of the maximal lactate steady state. Can J Appl Physiol 2002; 27: 232-249

22 MacLoughlin P, Popham P, Linton RAF, Bruce RCH, Band DM. Use of arterialised venous blood sampling during incremental exercise tests. J Appl Physiol 1992; 73: 937-940

23 Ribeiro L, Balikian P, Malachias P, Baldissera V. Stage length, spline function and lactate minimum swimming speed. J Sports Med Phys Fitness 2003; 43: 312-318

24 Simões HG, Denadai BS, Baldissera V, Campbell CSG, Hill DW. Relationships and significance of lactate minimum, critical velocity, heart rate deflection and $3000 \mathrm{~m}$ track-tests for running. J Sports Med Phys Fitness 2005; 45: 441-451

25 Smith CGM, Jones AM. The relationship between critical velocity, maximal lactate steady-state velocity and lactate turnpoint velocity in runners. Eur J Appl Physiol 2001; 85: 19-26

26 Smith MF, Balmer J, Coleman DA, Bird SR, Davison RCR. Method of lactate elevation does not affect the determination of the lactate minimum. Med Sci Sports Exerc 2002; 34: 1744-1749

27 Svedahl K, MacIntosh BR. Anaerobic threshold: the concept and methods of measurement. Can J Appl Physiol 2003; 28: 299-323

28 Tegtbur U, Busse MW, Braumann KM. Estimation of an individual equilibrium between lactate production and catabolism during exercise. Med Sci Sports Exerc 1993; 25: 620-627

29 Urhausen A, Coen B, Weiler B, Kindermann W. Individual anaerobic threshold and maximum lactate steady state. Int J Sports Med 1993; 14: $134-139$ 